\title{
Antimicrobial therapeutics delivery systems based on biodegradable polylactide/polylactide-co-glycolide particles
}

\author{
Robin Kumar ${ }^{1} \cdot$ Divya Jha $^{1} \cdot$ Amulya K. Panda ${ }^{1}$ (I)
}

Received: 19 February 2019 / Accepted: 24 February 2019 / Published online: 23 March 2019

(c) Springer Nature Switzerland AG 2019

\begin{abstract}
Infectious diseases are globally associated with high mortality in spite of the availability of therapeutic agents against most pathogenic microorganisms. This is due to the emergence of new infectious diseases and novel pathogen strategies to evade host defenses. There is thus a need to develop potent therapeutics and techniques for effective delivery of vaccines and drugs. Particles based on poly(lactic acid) and poly(lactic-co-glycolic acid) polymer-based particles are suitable delivery systems due to their biodegradable and biocompatible nature. They can be tailored to display various properties such as sustained release, dose sparing, bioactivity maintenance and targeted delivery. This review focuses on polymeric particle-based delivery systems to develop novel vaccines or drugs. Cellular interactions of particulate systems and the mechanism of action in animal models are also discussed.
\end{abstract}

Keywords Infectious diseases $\cdot$ Therapeutic agents $\cdot$ Biodegradable polymer $\cdot$ Polymeric particle $\cdot$ Delivery system

\section{Introduction}

Poly(lactic acid) and poly(lactic-co-glycolic acid) are food and drug administration approved biodegradable and biocompatible polymers used for various applications in human healthcare (Peres et al. 2017). The development of polylactide/polylactide-co-glycolide formulations as potential antigen/drug carriers emerges from the fact that these polymeric particles can be tuned in multiple ways to deliver the cargo selectively to specific sites and at controlled rates (Fig. 1). The mechanical properties of these polymers can be modified to develop efficient delivery system for different biomedical applications (Anderson and Shive 2012; Danhier et al. 2012) and enormous amounts of literature support that the polylactide/polylactide-co-glycolide polymers have extensive applications for entrapping bioactive molecules such as antigens, drugs, antibiotics. (Mitragotri et al. 2014). Polymers of different molecular weights can be used to

Robin Kumar and Divya Jha have contributed equally to this work.

Amulya K. Panda

amulya@nii.ac.in

1 Product Development Cell-II, National Institute of Immunology, Aruna Asaf Ali Marg, New Delhi 110067, India fabricate nano- or microparticles, and these particles show good stability upon storage and when administered in vivo. Since the release profiles of particles can be easily modulated, long-circulating polymeric particles are synthesized which are capable of releasing the drug at the site of action and possess desirable release kinetics can be synthesized. Particle properties can also be tailored accordingly by controlling the degradation rates or size, porosity, etc. (James et al. 2016).

Particle properties play a very important role in governing their interaction with the cells. Bulk and surface properties of these particles can also be changed to achieve desirable characteristics. Bulk properties are primarily changed by using polylactide/polylactide- $c o$-glycolide with other polymers. Surface properties can be modulated by changing the surface coating of polymer particles. This approach can be used to attain targeted delivery of these particles at specific sites. Entrapment efficiency, drug/antigen load and release profiles of these particles can be easily modified as per the requirements. Modifications such as pegylation of these polymeric particles can add to the stability of the entrapped bioactive molecule. Properties such as size and shape of particles can be altered to attain optimum pharmacokinetic and biodegradable profiles. The large surface to volume ratio of these particles provides more reaction sites to interact with the surrounding 


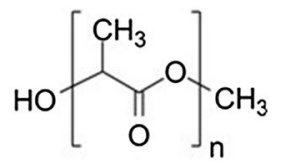

Polylactic acid<smiles>CC(C)OC(=O)C(C)(C)C(C)(C)OC(=O)C(C)O</smiles>

Polylactic-co-glycolic acid

\section{Targeting moiety}

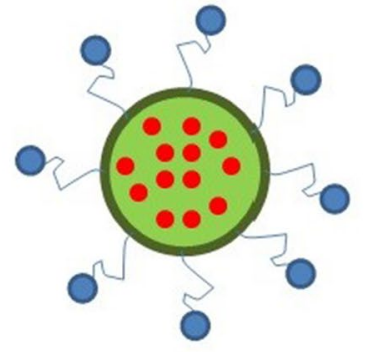

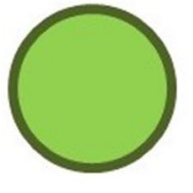

Polymeric particle

- Bioactive molecules
Fig. 1 Chemical structure of polylactic acid and polylactic-co-glycolic acid along with an indication of polymeric particle formulation to deliver a different kind of bioactive molecules. The chemical structure of these particles allows the flexibility of tailoring the functional

environment (Coelho et al. 2010). Owing to their safety profiles, biocompatibility, biodegradability and the ease with which the particle properties can be changed for a particular application, these polymers are gaining attention for a plethora of applications (Murariu and Dubois 2016).

The huge burden of infectious agents on human population and the high demand of therapeutic molecules against these diseases need an effective solution. Biocompatible polymeric particle entrapping bioactive molecules such as proteins, peptides, and antibiotics are capable of releasing these molecules over a desired period of time thereby decreasing the frequency of their administration (Pagels and Prud'homme 2015). Protection of bioactive molecules from degradation and enhancing the bioavailability of these molecules for a long time are additional advantages for considering it as a delivery system. Having these properties, polylactide/polylactide-co-glycolide particles also provide a great platform to be explored as adjuvants for development of new vaccines that can enhance the immune response of specific antigens against various diseases without compromising their safety (Gutjahr et al. 2016). This review reports different techniques to make polymer-based particle formulation, the cellular interaction of these formulations and recent advancement in their application for delivering bioactive molecules against microbes.

This article is an abridged version of the chapter published by Kumar et al. (2019) in the series Environmental Chemistry for a Sustainable World (https://www.springer. com/series/11480). groups on the surface, which subsequently brings about changes in the particle property. This can facilitate modulation of the particle properties for specific end application of these particles

\section{Preparation of polylactide/ polylactide-co-glycolide particles}

Different methods are employed to synthesize nanoparticles and microparticles from polymers such as poly(lactic acid) and poly(lactic-co-glycolic acid). Double emulsion solvent evaporation is a commonly used method to encapsulate bioactive molecules into particles. The aqueous phase containing the water-soluble compound is added to the polymer containing organic phase to create primary w/o emulsion. This emulsion is then added to the aqueous phase containing stabilizers to create a w/o/w double emulsion. Solvent evaporation then yields particles. Antibiotics like ciprofloxacin and other novel antimicrobial peptides have been entrapped into polylactide-co-glycolide nanoparticles using this method to check biofilm development (Cruz et al. 2017; Thomas et al. 2016). Double emulsion solvent evaporation method allows tailoring the particle properties in endless ways, exploiting the different variables influencing the process.

Nanoprecipitation (also known as solvent displacement method) is a simple one-step process for the preparation of polylactide-based nanoparticles and requires two miscible solvents. An important prerequisite is that both the polymer and the bioactive molecule must be soluble in one (same) solvent but should not be soluble in the second one. When the polymer-drug containing solvent diffuses into the second solvent which is present in an excess amount, sudden desolvation of the polymer allows entrapment of bioactive molecule inside the polymeric particles. If the process parameters are kept constant, consistent batches of particles can be produced using nanoprecipitation (Sahin et al. 2017). Using this method, anticancer drugs have been successfully entrapped using nanoprecipitation with high 
drug load (El-Hammadi et al. 2017; Pandey et al. 2016). Tamoxifen was entrapped into polylactide-co-glycolide nanoparticles using nanoprecipitation, and an entrapment efficiency as high as $71 \%$ was observed.

Other methods like spray drying, microfluidic technique, and template-/mold-based method are now being used for more uniform particles and large-scale production. Spray drying is a single step process of transforming a liquid feed into a dry form by passing the liquid through a medium (inert gas such as nitrogen) (Wan and Yang 2016). It is a useful, scalable particle formulation method, where an organic phase containing drug dissolved or dispersed in a polymer is sprayed as ultra-fine droplets in dry air flow. Microspheres entrapping antimicrobial drugs such as cefquinome sulfate can be synthesized using spray drying and has been shown to be targeted to the lungs where they showed a sustained release of the drug in vivo (Qu et al. 2017). Microfluidics-based devices are used to produce uniform particles with precisely controlled release profiles. Polymers are dissolved in organic solvents and single/ double emulsions are made in microfluidic devices, by droplet solidification through solvent evaporation (Ekanem et al. 2017b). Bipolymer microparticles have also been synthesized using microfluidic emulsification and solvent evaporation using poly(lactic acid) and polycaprolactone (Ekanem et al. 2017a). Using template-based methods, gels can be made that can change their sol-gel phase upon receiving their specific cue such as temperature. The warm gel solution is put in the hard cast. Once the mold solidifies, it is extracted from the mold and the solvent is evaporated (Basu et al. 2016; Saffer et al. 2011). The advantages associated are reproducibility in the batches, monodispersed particle formation with high drug load. Another variant of the template-based method is molecular imprinting which imparts a 'memory' to the polymer. This is an emerging technology and has to be exploited to yield maximal use of the approach (Gagliardi et al. 2017).

Several parameters can influence these processes of particle making such as the amount of compound to be entrapped, initial polymer concentration, type of solvent, surfactant concentration, the ratio of aqueous phase to organic phase, stirring rate and other variables. The parameters that affect the particle formation also include the injection rate of the organic phase into the anti-solvent, the compound: polymer ratio, solvent evaporation rate and the solubility of the compound. The choices of a combination of the drug/polymer and solvent/ nonsolvent system are yet other important parameters.

\section{Different facets of an interaction of the polymeric particles with the cells}

The cells can internalize polymeric particles of sub-cellular size range and the interaction between particles and cells are influenced by particle properties. The cells can take up these particles by endocytosis, which is a broad term that encompasses several energy-dependent mechanisms such as phagocytosis, pinocytosis, macropinocytosis, and receptor-mediated endocytosis (Zhao et al. 2011) (Fig. 2). Exocytosis extrudes a major proportion of the internalized particles out. A very small fraction of the remaining internalized fraction escapes the endosomal compartment and provides the therapeutic benefits owing to particle retention in the cytosol (Panyam and Labhasetwar 2003; Panyam et al. 2002). Hence, for the maximal efficacy of a drug delivery particulate system, the mode of uptake of these particles becomes an important concern (Hillaireau and Couvreur 2009).

To enhance the internalization of polylactide/polylactide-co-glycolide nanoparticles inside the cells, different factors are taken into accounts such as size, shape, charge, and ligands (Saraiva et al. 2016) (Fig. 3). Particle properties such as size and shape influence their cellular uptake (Roointan et al. 2018; Zhao et al. 2011). The particle size of around $500 \mathrm{~nm}$ is found to be optimal for efficient phagocytosis of these particles (Hillaireau and Couvreur 2009). It is also reported that charged particles either positive or negative have better internalization into the cells than the neutrally charged polymeric particles (Fröhlich 2012; Hillaireau and Couvreur 2009). It is also observed that the mode of entry of polylactide particles inside the cells may vary depending on their surface being cationic or anionic (Fröhlich 2012). Polylactide-co-glycolide nanoparticles also find their use in treating inner ear drug delivery. Modulating the sizes of these particles and their surface modification using polyethylene glycol/chitosan have shown to enhance drug delivery to the inner ear (Cai et al. 2017).

Surface modification of the particles has also a great impact in affecting biodistribution; cellular uptake and endosomal escape of these particles (Vasir and Labhasetwar 2007). Surface functionalization of particles can be used to target dual cellular populations (Roointan et al. 2018). The surface charge of the pegylated polylactide nanoparticles is seen to also affect the oral bioavailability of particles after their administration (Du et al. 2018). Pegylation of the nanoparticles is a commonly used approach to confer hydrophobicity on the particles and hence enhance the interaction with the cells (Aldrian et al. 2017; Suk et al. 2016). Surface decoration of the ligands to specific markers or receptors on the surface of the cells can improve the particle uptake by the cells. One of the approaches to enhance the internalization involves the surface modification of these particles by cell-penetrating peptides which are hydrophobic and cationic (Steinbach et al. 2016). Peptides being smaller in size, lower immunogenicity and with ease of synthesis serve as good targeting molecules serve good for improving the bioavailability of 
Fig. 2 Different modes of cellular uptake of polymeric nano- and microparticles. The cells, in multiple ways, can take up polymeric nanoparticles such as phagocytosis, pinocytosis, micropinocytosis, and receptormediated endocytosis. Particle properties play a major role in governing the mode of uptake of these particles into the cells. Physical properties such as size and shape and chemical modifications on the surface affecting the surface charge or hydrophobicity of these particles have a huge impact in mediating the interaction of these particles with the cells
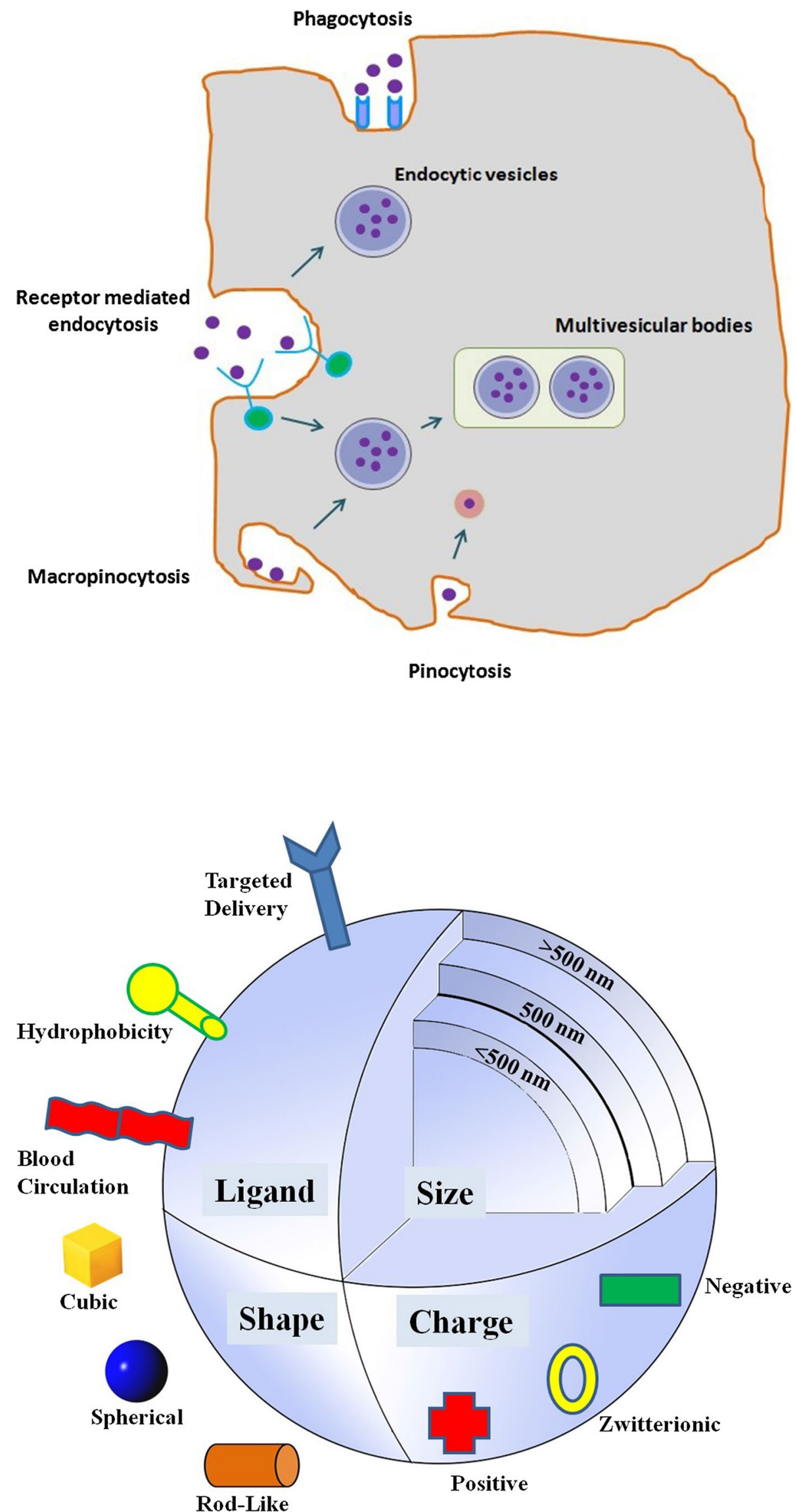

Rod-Like
Phagocytosis

Pinocytosis
Fig. 3 Factors influencing the polymeric particle-mediated bioactive delivery: different sized particle can be formulated for efficient delivery of bioactive molecules. Shape (spherical, cubic, and rod-like) and charge (negative, zwitterionic, and positive) can be varied to enhance entrapment and cellular interaction; poly(lactic-co-glycolic acid) and poly(lactic acid) particles are negatively charged. Surface functionalization of a particle is another important parameter that can influence the bioactive delivery; ligands such as poly(ethylene glycol) improve particle presence in circulation, amphiphilic peptide increases hydrophobicity and receptors/antibodies help in targeted delivery. Adapted from (Saraiva et al. 2016) 
poorly adsorbed drugs (Gourdon et al. 2017). Sun et al. 2017 reported designing of abalone peptide functionalized polylactide nanoparticles entrapping doxorubicin and showed that both tumor cells and vascular endothelial cells took up these particles more efficiently. This may be a very promising approach for the development of anticancer drug delivery systems.

Stabilizers used during the particle synthesis may be used to target ligand coupling on the particle surface, which ultimately influences the internalization of these particles into the cells. Raudszus et al. 2018 reported the use of a stabilizer vinyl sulfone-modified poly(vinyl alcohol)-derivative to prepare polylactide nanoparticles which showed different uptake behavior in cerebral endothelial cells owing to different functional groups present on the surface of these particles. Thus, specific stabilizers may be used for specific targeting applications.

In case of many diseases, direct antigen delivery is not practically useful because of the limitations such as low immunogenicity, low stability as well as the lesser half-life of these antigens in vivo. Polymeric particles offer solutions to these limitations and are emerging as promising delivery systems in immunotherapy (Tran et al. 2018). Using polymeric particles not only allow slow and sustained release of antigens after their administration, but also allow targeted delivery of these particles, incorporation of multiple components in a single assembly, enhancing uptake as well as modulating the immune response (Silva et al. 2016). It is observed that antigen entrapped into polylactide-co-glycolide nanoparticles, when immunized into the animals, could elicit the higher antigen-specific immune response than the particles that had antigens adsorbed upon the particles (Liu et al. 2016). Using polymeric particles, offer the ease of tailoring the particle properties to meet specific enduse application.

\section{Polylactide/polylactide-co-glycolide particulate delivery system of bioactive therapeutics}

Among the polymeric delivery system, biodegradable and biocompatible polylactide/polylactide-co-glycolide-based particulate system have been extensively investigated for therapeutic bioactive molecules. Biodegradable polymeric particulate system can be used as a delivery system and has an ability to entrap and present different bioactive molecules of interest on their surface. However polymeric particles have many challenges to overcome such as low entrapment efficiency, low loading capacity and product sterility for animal use. The recent development of a novel or improved method for bioactive molecule-loaded polymeric particle formulation will lead to the successful application of polymeric particle-based delivery system. The size of polymeric particles can modulate the immune response against antigen delivered through particles (Oyewumi et al. 2010; Shah et al. 2014). Depending on the required immune response or effective drug treatment against the specific infectious agent, different sized particles can be formulated to deliver therapeutic molecules (Fig. 4). Several new polylactide/polylactide-co-glycolide-based delivery systems have now been developed and showed great promise for various kinds of bioactive molecules alone and in combination to overcome the global problem of infectious diseases (Table 1).

\section{Nanoparticle-based delivery system}

In recent times, biocompatible polymeric nanocarriers have been widely explored as a delivery system for bioactive molecules such as an antigen, nucleic acid, antibiotics. These nanocarriers help them to sustain for longer duration in the host system and avoid getting degraded rapidly. Considering this, polylactide and polylactide-co-glycolide nanoparticles entrapping GIBIM-P5S9K peptide were formulated using double emulsion method (Cruz et al. 2017). GIBIM-P5S9K peptide a new antimicrobial molecule inhibited the growth of Pseudomonas aeruginosa, Escherichia coli O157:H7 and methicillin-resistant Staphylococcus aureus at very low peptide concentration when it was delivered using nanoparticle. It enhanced the antimicrobial activity by 20 fold compared to the soluble peptide. In vivo study also suggested that these particles are hemocompatible. In another study, Türeli et al. 2017 showed that cystic fibrosis lung infected with $P$. aeruginosa can also be treated using antibiotic-loaded polylactide-co-glycolide nanoparticles. Ciprofloxacin-loaded nanoparticles were able to penetrate the barrier of biofilm/mucus and showed enhanced antimicrobial activity compared to ciprofloxacin complex. These nanocarriers were nontoxic to human epithelial cell lines at a minimum inhibitory concentration of antibiotic required against bacteria. It could be a new strategy to treat $P$. aeruginosa infections in cystic fibrosis lung.

For making an effective delivery vehicle Pei et al. (2017), formulated a nanoparticle delivery system using a blend of polymers with distinct features for vancomycin against Methicillin-resistant $S$. aureus infection. Vancomycin is ineffective against this intracellular pathogen due to poor cellular uptake. Considering this limitation, four polymers were used for nanoformulation: polylactide-co-glycolide as a main delivery system, polyethyleneglycol-polylactide-coglycolide conjugate for maintaining vancomycin polarity, Eudragit E100 for enhancing entrapment and a chitosan derivative for $\mathrm{pH}$-sensitive drug release. The result showed that this nanoparticle was effective against intracellular pathogens than free vancomycin and could be more promising 


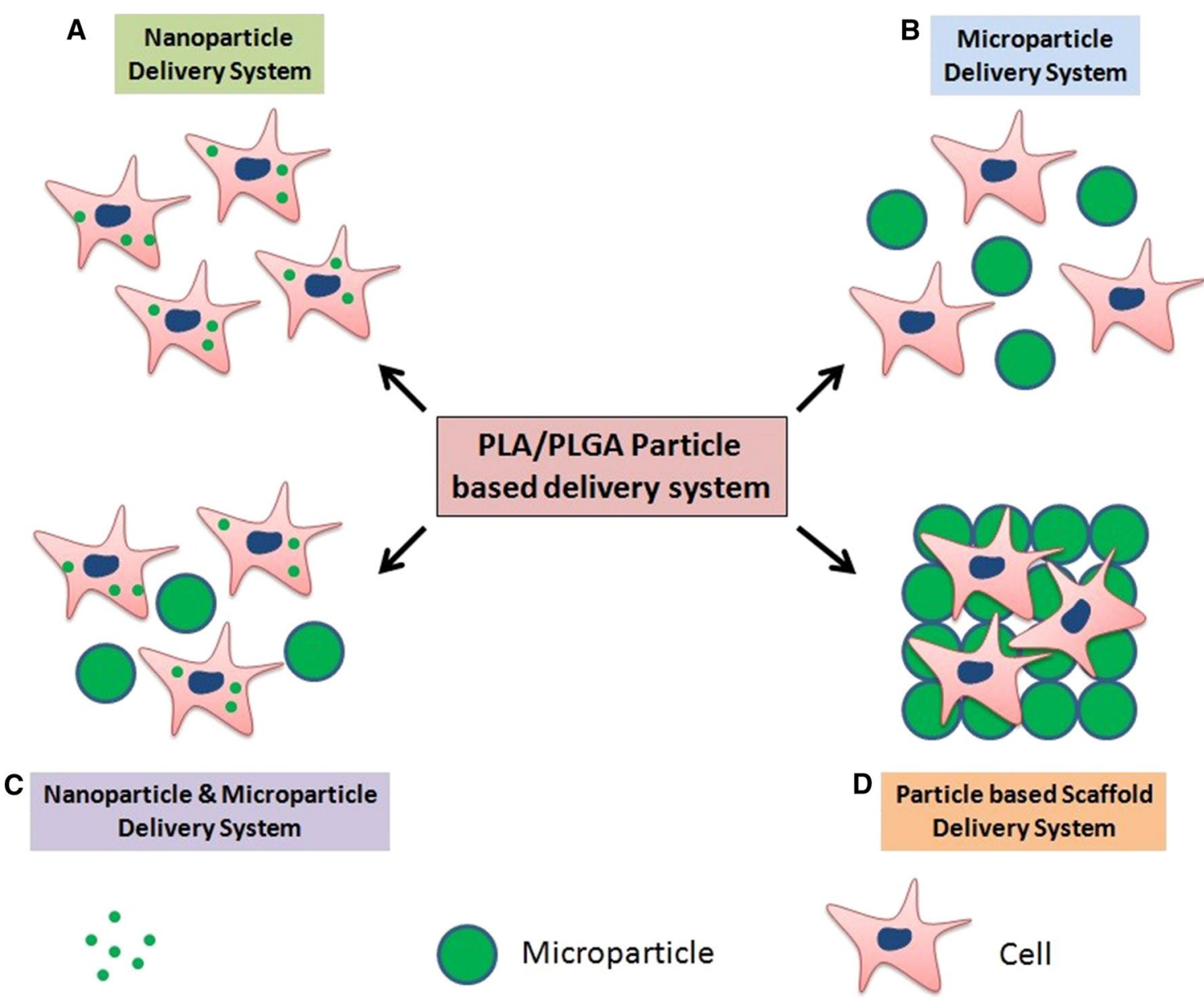

Fig. 4 Schematic illustration of various approaches for polylactide/ polylactide-co-glycolide particle-based delivery system. a Nanoparticle-based delivery system. b Microparticle-based delivery system. c Nanoparticle and Microparticle-based delivery system. d Particlebased Scaffold delivery system. Nanoparticle and microparticle-based

for treatment. Tan et al. (2017) has also shown the role of hypromellose phthalate HP55/polylactide-co-glycolide nanoparticle as an oral delivery system for $\mathrm{H}$. pylori recombinant antigen CCF; a combination of a multi-epitope vaccine CTB-UE and a chimeric flagellum that protects antigen from the gastrointestinal environment. Also immunized mice with these nanoparticles induced urease-specific antibody and $\mathrm{T}$ cell-mediated response. Moreover, immunized mice were protected after $H$. pylori challenge. These results indicated that use of these nanoparticles as antigen delivery system for gastrointestinal infection could be a promising strategy. In the recent study, Seok et al. (2017) designed a influenza $A(H 1 N 1) H 1 N 1$ vaccine delivery system using polyplexes of polylactide-co-glycolide/polyethylenimine nanoparticles coated on microneedle. This system was optimized for high transfection efficiency in mammalian cells. Intradermally immunized mice induced enhanced humoral immune response than an intramuscular delivery methods are used for years but have seen recent advancements in their applications owing to our greater understanding of these methods. Also, newer methods have come up that take care of the limitations associated with the older methods, allowing a larger range of use of these particles. PLA/PLGA polylactide/polylactide- $c o$-glycolide

of polyplex containing H1N1 deoxyribonucleic acid using microneedles. However, immunogenicity against H1N1 protein was still weak therefore such systems need to be improved for a desired immune response against influenza virus. A deoxyribonucleic acid vaccine coated on the surface of polylactic-co-glycolic acid-polyl-lysine/poly- $\gamma$-glutamic acid nanoparticles was formulated for Ebola vaccination using microneedle patch (Yang et al. 2017). Nanoparticles were administered in mice through intramuscular or with microneedle patch to skin. Although antibody titer value was not significantly different for both routes, neutralizing activity of antibody against Ebola virus was higher after microneedle immunization. That concluded the use of polylactic-co-glycolic acid-polyl-lysine/poly- $\gamma$-glutamic acid nanoparticles may be needed for development of a robust and immunogenic microneedle patch for Ebola vaccine.

Food and drug administration approved biodegradable and biocompatible polylactide and polylactide-co-glycolide 
Table 1 Polylactide/polylactide-co-glycolide-based delivery system against various infectious agents

\begin{tabular}{|c|c|c|c|c|}
\hline Bioactive delivery system & Key components & Bioactive molecules & Related microorganism/disease & References \\
\hline \multirow[t]{17}{*}{ Nanoparticle } & PLA and PLGA & GIBIM-P5S9K peptide & $\begin{array}{l}\text { P. aeruginosa, E. coli O157:H7 } \\
\text { and Methicillin-resistant } S . \\
\text { aureus }\end{array}$ & Cruz et al. (2017) \\
\hline & PLGA & Ciprofloxacin & P. aeruginosa & Türeli et al. (2017) \\
\hline & $\begin{array}{l}\text { PLGA } \\
\text { PEG-PLGA } \\
\text { Eudragit E100 } \\
\text { ZWC }\end{array}$ & Vancomycin & Methicillin-resistant $S$. aureus & Pei et al. (2017) \\
\hline & HP55/PLGA & $\mathrm{CCF}$ & Helicobacter pylori & Tan et al. (2017) \\
\hline & PLGA/PEI & H1N1 DNA vaccine & $\mathrm{H} 1 \mathrm{~N} 1$ virus & Seok et al. (2017) \\
\hline & PLGA-PLL $/ \gamma$ PGA & Ebola DNA vaccine & Ebola virus & Yang et al. (2017) \\
\hline & PLGA & CNA19 & S. aureus & (Genta et al. (2016) \\
\hline & PLGA & HA, NP, PA, and M2e-PP peptide & H1N1 virus & Hiremath et al. (2016) \\
\hline & PLGA & $\mathrm{KAg}$ & Swine influenza virus & Dhakal et al. 2017) \\
\hline & PLGA & LCP-1 & Group A Streptococcus & Marasini et al. (2016) \\
\hline & PLGA & rROP18 & Toxoplasama gondii & Nabi et al. (2017) \\
\hline & PLGA & Dengue virus E-protein & Dengue virus & Metz et al. (2016) \\
\hline & PLGA & TLR7 agonist and DTaP vaccine & Diphtheria, tetanus and pertussis & Bruno et al. (2016) \\
\hline & PLGA & $\begin{array}{l}\text { sLiAg, MPLA, TNF } \alpha \text { mimicking } \\
\text { peptide }\end{array}$ & Leishmaniasis & Margaroni et al. (2016) \\
\hline & PLGA & CPA160-189, MPLA & Leishmania infantum & Agallou et al. 2017) \\
\hline & PLGA & $\begin{array}{l}\text { Envelope gp140, Gag p55 protein, } \\
\text { TLR4 and TLR7/8 agonists }\end{array}$ & Simian immunodeficiency virus & Kasturi et al. (2017) \\
\hline & PLA & FNIII $9 / 10$ protein and p24 & Human immunodeficiency virus & Dalzon et al. (2016) \\
\hline \multirow[t]{7}{*}{ Microparticle } & PLGA & Outer membrane proteins (porins) & Salmonella Typhi & Carreño et al. (2016) \\
\hline & PLGA & HCV-E2 & Hepatitis $\mathrm{C}$ virus & (Roopngam et al. (2016) \\
\hline & PLGA & rSAG1 and rGRA2 & T. gondii & Allahyari et al. (2016) \\
\hline & PLGA & rCDPK6 and rROP18 & T. gondii & Zhang et al. (2016) \\
\hline & PLGA & Inactivated polio vaccine & Polio virus & (Tzeng et al. (2016) \\
\hline & PLGA & rOMVs & Influenza A virus & Watkins et al. (2017) \\
\hline & PLGA & NH36 and $3 \mathrm{M}-052$ & L. donovani & Wang et al. (2017) \\
\hline Nanoparticle/microparticle & PLGA & Pertussis toxoid & Pertussis & Li et al. (2016) \\
\hline Scaffold & PLA and PLGA & Gentamicin, Neomycin & S. aureus & Admane et al. (2017) \\
\hline
\end{tabular}

PLA poly(lactic acid), PLGA poly(lactic-co-glycolic acid), PEG polyethyleneglycol, ZWC zwitterionic chitosan, HP55 hydroxypropylmethyl cellulose phthalate, $C C F$ a construct comprising a dual-antigen epitope and dual-adjuvant vaccine, PEI polyethylenimine, H1N1 an influenza A virus, DNA deoxyribonucleic acid, $P L L / \gamma P G A$ poly-l-lysine/poly- $\gamma$-glutamic acid, CNA19 a purified recombinant collagen-binding bacterial adhesion fragment, HA, NP, PA, and M2e-PP peptide, conserved peptides of influenza a virus, $K A g$ inactivated swine influenza virus H1N1 antigens, $L C P-1$ Lipid core peptide, $r R O P 18$ recombinant rhoptry protein $18, D T a P$ diphtheria-tetanus-pertussis, sLiAg soluble Leishmania antigens, TNF $\alpha$ tumor necrosis factor alpha, CPA160-189 cysteine protease A, MPLA monophosphoryl lipid A, FNIII9/10 human fibronectin recombinant proteins, $p 24$ an HIV antigen, $H C V$-E2 E2 envelope glycoprotein of hepatitis $\mathrm{C}$ virus, $r S A G 1$ and $r G R A 2$ recombinant surface antigen 1 and recombinant dense granular protein, $r C D P K 6$ recombinant calcium-dependent protein kinase 6, rOMVs recombinant outer membrane vesicles, NH36 Leishmania donovani nucleoside hydrolase antigen, 3M-052 a toll-like receptor7/8 agonist

polymer have gained great attention for vaccine development due to its ability to modulate immune response specific to the antigen. Genta et al. (2016) showed the adjuvant property of polylactide- $c o$-glycolide-based nanoparticle for a purified collagen-binding fragment (CNA19 peptide) from S. aureus. In vivo study in mice showed that polymeric nanoparticle has more potential as an adjuvant with respect to alum for subcutaneous route of CNA19 immunization. Furthermore study proved that compare to intranasal route, subcutaneous immunization will be the preferential route for developing CNA19-loaded nanoparticle vaccine. In another study, Hiremath et al. (2016) developed polylactide-co-glycolide nanoparticle delivery system for influenza virus conserved peptides. Influenza virus conserved peptides are potentially able to elicit the cross-protective immune response but are poorly immunogenic. Pigs immunized with nanoparticle entrapped peptides induced $\mathrm{T}$ cell-specific response, not enhanced antibody response. Challenge with virulent influenza virus 
showed no fever and flu symptoms in nanoparticle-loaded peptides immunized pig. In summary, polymeric nanocarrier could be effective to develop a vaccine with high $\mathrm{T}$ immune response. In a similar way, Dhakal et al. (2017) formulated a polylactide-co-glycolide nanoparticle entrapped inactivated swine influenza virus H1N1 antigens formulation against influenza flu. Pigs immunized with antigen-loaded nanoparticle induced strong antigen-specific $\mathrm{T}$ cell response and had no flu symptoms after challenge with heterologous swine influenza virus. In summary, inactivated influenza virusloaded nanoparticle can be a solution for making influenza virus vaccine. Recently, Marasini et al. (2016) also designed a polylactide- $c o$-glycolide nanoparticle-loaded lipopeptide vaccine to prevent Group A Streptococcus infections. Study showed that lipopeptide entrapped nanoparticle improved uptake of antigen in antigen presenting cells and enhanced immunoglobulin (Ig) level; mucosal IgA and systemic IgG response against lipopeptide. That helps in bacterial growth inhibition and clearance.

Nabi et al. (2017) showed a noninvasive approach for developing polylactide-co-glycolide-based vaccine against Toxoplasma gondii. The nanocarriers were formulated to encapsulate recombinant rhoptry protein (rROP18) and administered in mice through intranasal immunization. Immunized mice elicited enhanced $\operatorname{IgG} 2 \mathrm{a}$ and $\operatorname{IgA}$ response compared to control. However immunological study still needs to be explored to translate these findings into a potential vaccine. New method like particle replication in nonwetting template (PRINT) technology was also explored to make effective subunit vaccine against infectious agents. Development of dengue vaccine is tricky because the vaccine should protect against all four infectious dengue virus serotypes. Several vaccine formulations including live attenuated tetravalent virus vaccine showed only partial efficacy. Considering this limitation, Metz et al. (2016) used the PRINT technology to form dengue virus E-protein adsorbed nanoparticles. Immunization study showed that nanovaccine induced higher IgG that was more effective compared to soluble antigen protein. These results indicate that polylactide-co-glycolide nanoparticles using PRINT technology seems promising platform for subunit vaccine delivery.

Further enhancement in its adjuvant property needs incorporation of immunepotentiator molecules like toll-like receptor (TLR) agonists. More advanced polymeric delivery systems have been developed to incorporate both antigen and immunepotentiator. This system can deliver both antigen and immunepotentiator together at the particular site. Bruno et al. (2016) combined the adjuvant role of polylactide-coglycolide nanoparticle with a synthetic immune potentiator molecule that targets TLR7. Double emulsion method was used to formulate nanoparticle entrapped TLR7 agonist and then diphtheria-tetanus-pertussis (DTaP) vaccine was adsorbed onto the nanoparticles. To see the adjuvanticity of this nanoformulation, mice model was used and evaluated for the improvement in the efficacy of DTaP vaccine. codelivery of TLR7 agonist through nanoparticle and antigens resulted in high IgG and IgG2a antibody titers compared to nonentrapped form. That concluded that polylactide- $c o-$ glycolide can be used along with other immunomodulators to get the desired immune response against antigens. In another study, soluble Leishmania antigens and monophosphoryl lipid A adjuvant entrapped polylactide- $c o$-glycolide nanoparticles were formulated with tumor necrosis factor alpha mimicking peptide on their surface (Margaroni et al. 2016). Efficient uptake of nanoparticles by dendritic cells helped in inducing its maturation and differentiation. That resulted in high level of the co-stimulatory molecules and stimulated the production of interleukins; IL-12 and IL-10 cytokines. nanoparticles primed dendritic cells promoted $\mathrm{T}$ cell activation and differentiation which was characterized by $\mathrm{T}$ cell-specific transcriptional factors and cytokine expression. These results indicated that this new nanoformulation could be considered as a potential vaccine candidate against Leishmaniasis. Similarly, a peptide-based polylactide-co-glycolide nanovaccine was also reported to enhance the immunogenicity against Leishmaniasis (Agallou et al. 2017). Cysteine protease A (CPA160-189), a synthetic peptide containing overlapping epitopes of a highly immunogenic Leishmania protein, was entrapped along with monophosphoryl lipid A adjuvant in polylactide-coglycolide nanoparticles. In vivo study in the visceral Leishmaniasis susceptible BALB/c mice model showed a significant reduction in parasite burden compared to control group. Suggesting that a well-designed peptide-loaded nanocarriers could be a promising vaccine candidate against Leishmaniasis. Recently, Kasturi et al. (2017) investigated the adjuvant role of polylactide-co-glycolide nanoparticles containing TLR4 and TLR7/8 agonists for envelope gp 140 and Gag p55 protein against simian immunodeficiency virus (SIV); SIVmac239. This formulation was immunized with virus-like particles containing gp140 and Gag p55 in Rhesus macaques. Nanoparticles adjuvanted formulation induced robust immune response and showed high protection against intra-vaginal challenges with heterologous strain of SIV in animals. Such formulation can also be designed for other viral infection.

Targeted delivery of antigens is one of the major concerns to make an effective vaccine. In order to develop an efficient vaccine delivery system for an HIV antigen (p24), Dalzon et al. (2016) developed a human fibronectin protein (FNIII9/10) coated polylactide nanoparticle system to target an integrin $\alpha 5 \beta 1$ positive cells. Human fibronectin FNIII9/10 proteins have an integrin $\alpha 5 \beta 1$ binding site, the RGDS sequence (Arg-Gly-Asp-Ser) that enhanced cellular uptake of nanoparticles by $\alpha 5 \beta 1$ positive cells. Subcutaneously immunized mice with nanoparticles co-coated with 
p24, and FNIII9/10 proteins showed no significant improvement in humoral response compared to nanoparticles coated with p24. However, the presence of FNIII9/10 proteins helped in increasing avidity index of p24 antibody.

\section{Microparticle delivery system}

Polylactide/polylactide-co-glycolide microparticle can be very useful for entrapping high concentration of bioactive molecule. Moreover, it can be used for the long and sustain delivery of therapeutic molecules in blood circulation. These microparticles provide thermostability and maintain the nature of loaded bioactive molecules such as antigenicity of a vaccine, bioactivity of antibiotic. Currently, there are two vaccines available for Salmonella Typhi infection but both are thermolabile. Carreño et al. (2016) formulated the S. Typhi outer membrane protein (porin) entrapped polylactide-co-glycolide microparticle for making a thermostable oral vaccine. An orally administered vaccine was protected from an acidic environment and showed strong B cell immune response in mesenteric lymph nodes and Peyer's patches. In general, microparticle encapsulation improved the efficacy of S. Typhi oral vaccine.

Microparticles not only protect antigen degradation in circulation but also help in the modulation of an immune response against antigens. Recently, Roopngam et al. (2016) reported $\mathrm{E} 2$ envelope glycoprotein of hepatitis $\mathrm{C}$ virus type (HCV-E2)-loaded polylactide-co-glycolide microparticle as a potential vaccine against hepatitis $\mathrm{C}$ virus. This virus causes hepatitis and liver carcinoma and there is need to develop a vaccine. HCV-E2 helps the virus to bind with host cells and antibody against it is very effective in neutralizing the virus. Results suggested that vaccinated animals had the high number of CD8 + T-cells and Interferon gamma (IFN $\gamma$ ) secreting immune cells. Moreover, the antigen-specific antibody titer was also high for HCV-E2 microparticles. Thus antigen delivery using microparticle can be a good strategy for a vaccine development. Polylactide-co-glycolide microparticle was also used to design protein-based subunit vaccine against $T$. gondii using a recombinant surface antigen 1 (rSAG1) and recombinant dense granular protein (rGRA2) (Allahyari et al. 2016). rSAG1 and rGRA2 adsorbed microparticles were used to enhance the immune response in $\mathrm{BALB} / \mathrm{c}$ mice. All immunized mice were able to induce strong humoral and cellular response compared to control. rSAG1 and rGRA2 microparticles vaccinated mice were also able to survive for longer times and partially protected against acute toxoplasmosis compared to a single antigen. Similarly, Zhang et al. (2016) developed the recombinant calcium-dependent protein kinase 6 (rCDPK6) and rhoptry protein 18 (rROP18) entrapped polylactide- $c o$-glycolide microparticle for generating long-lasting immune response against $T$. gondii. Mice immunized with this formulation showed T helper cell type 1(Th1) response with enhanced IFN $\gamma$ and IL-2 cytokines and specific antibodies response. Also vaccinated mice conferred high protective immunity after $T$. gondii challenge compared to control. These studies hold the great promise of polymeric microparticle as a delivery system in the development of a vaccine against $T$. gondii.

Microparticle delivery system has also been investigated for a single shot vaccine development. In order to resolve the requirement of multiple doses of inactivated polio vaccine (IPV) to obtain full protection, Tzeng et al. (2016) developed IPV entrapped polylactide-co-glycolide microparticle. Rat immunized with IPV entrapped microparticle showed robust and long-lasting antibody response compared to multidose soluble IPV. This technique can be used to eradicate infectious diseases such as polio using single dose vaccine. For influenza A virus, Watkins et al. (2017) described a single dose polylactide-co-glycolide microparticle entrapping recombinant outer membrane vesicles (rOMVs) which has the heterospecies tandem sequence of the M2 protein. Immunized $\mathrm{BALB} / \mathrm{c}$ mice elicited high IgG titers and produced the high level of IFN $\gamma$ for rOMV microparticle. After challenge with lethal dose of influenza virus, vaccinated mice were also protected against influenza. These data support the role of polylactide-co-glycolide microparticle for making a single shot vaccine against influenza.

The role of additional adjuvant has also been evaluated along with polymeric particle to improve the efficacy of potential vaccines. In a recent study, Wang et al. (2017) formulated polylactide- $\mathrm{co}$-glycolide microparticle co-entapping a 36-kDa nucleoside hydrolase recombinant protein antigen (NH36) from Leishmania donovani along with the TLR7/8 agonist 3M-052 to generate Th1 immune response against Leishmaniasis. NH36 and 3M-052-loaded particles elicited enhanced Th1 response-associated $\operatorname{IgG} 2 \mathrm{a}$ and $\operatorname{IgG} 2 \mathrm{~b}$ antibodies and have more IFN $\gamma$ producing splenocytes compared to other groups in BALB/c mice. These results indicate that polymeric particles along with $3 \mathrm{M}-052$ could be used as an adjuvant for Leishmaniasis vaccines.

\section{Nanoparticle/microparticle delivery system}

Both nanoparticle and microparticle-based vaccine delivery system have been demonstrated for different immune modulation against antigen (Gregory et al. 2013; Johansen et al. 2000; Peyre et al. 2004). Nanoparticles are reported to promote cellular immune response whereas microparticle enhanced humoral response (Chong et al. 2005; Gutierro et al. 2002). Recently, Li et al. (2016) investigated polylactide-co-glycolide-based nanoparticle/microparticle as a vaccine delivery system for pertussis antigen. In vitro study showed higher uptake of antigen in macrophage cells for polymeric nano/microparticles and immunized mice significantly enhanced INF- $\gamma$ and IL-17 cytokines level in 
splenocytes after priming with heat-killed Bordetella pertussis, indicating a Th1/Th17 response. Also, vaccinated mice were protected against subsequent pertussis infection. This finding suggests that polylactide-co-glycolide nanoparticle/ microparticle may serve as an alternative to acellular pertussis vaccine for balanced Th1/Th2 immune response.

\section{Biodegradable polymeric scaffold}

Biodegradable and biocompatible polymers have now been explored for making particle-based fabrication of bioactive molecule-loaded self-assembled scaffold. It offers many advantages such as safety, desirable loading and sustained release of loaded molecules in comparison with other implanted delivery systems. This polymeric scaffold-based system is now serving as a platform for many applications such as localized delivery of antibiotics, wound healing and tissue engineering. Admane et al. (2017) described a new method to design gentamicin and neomycin-loaded scaffold using polylactide particles. Firstly antibiotics were loaded in polylactide during microparticle formulation and then these particles were fused using methanol to form scaffold at room temperature. The bioactivity of antibiotic from scaffold was tested and confirmed against $S$. aureus. The scaffold was nontoxic to mammalian cells and supported the attachment and proliferation of cells. Subcutaneous implantation of scaffold in mice demonstrated the cellular infiltration and vascularization in scaffolds. Thus, this study provides an easy method to design a polylactide-based scaffold for various applications.

\section{Conclusion}

Biopolymers are making their progress in a wide range of biomedical applications. It is expected that the suitability of these polymers is going to rocket up owing to endless ways of tailoring the particle properties to suit the desired application. Newer methods are coming up to design these polylactide/polylactide-co-glycolide nano- and microparticles for specific end-use properties. Blends are made using these polymers in combination with other polymers to overcome the limitations such as low glass transition temperature or fast degradation rate. Other natural and synthetic fibers are also used with these polymers. Thus, these polymers are seen as promising materials to suit a diverse range of possibilities in biomedical applications. Polylactide and polylactide-co-glycolide polymer slowly degrade and form lactic acid and glycolic acid as a degradation products which are components of various metabolic pathways in animal model (Brady et al. 1973). It makes these polymers more suitable for various biomedical applications including drug, antigen and antibiotic delivery system. Requirement of safe and reproducible vehicle, targeted delivery system, personalized medicine and delivery of specific bioactive molecule have led to the exploitation of biodegradable and biocompatible polylactide and polylactide-co-glycolide polymers.

Acknowledgements The authors are grateful to the National Institute of Immunology for financial support.

Authors' contributions Robin Kumar and Divya Jha have an equal contribution to this work, and all authors read and approved the final manuscript.

\section{Compliance with ethical standards}

Conflict of interests The authors declare that they have no conflict of interests.

\section{References}

Admane P, Gupta J, Ancy I, Kumar R, Panda AK (2017) Design and evaluation of antibiotic releasing self-assembled scaffolds at room temperature using biodegradable polymer particles. Int J Pharm 520(1):284-296. https://doi.org/10.1016/j.ijpharm.2017.01.071

Agallou M, Margaroni M, Athanasiou E, Toubanaki DK, Kontonikola K, Karidi K, Kammona O, Kiparissides C, Karagouni E (2017) Identification of BALB/c immune markers correlated with a partial protection to leishmania infantum after vaccination with a rationally designed multi-epitope cysteine protease a peptidebased nanovaccine. PLoS Negl Trop Dis 11(1):e0005311. https ://doi.org/10.1371/journal.pntd.0005311

Aldrian G, Vaissière A, Konate K, Seisel Q, Vivès E, Fernandez F, Viguier V, Genevois C, Couillaud F, Démèné H (2017) PEGylation rate influences peptide-based nanoparticles mediated siRNA delivery in vitro and in vivo. J Controll Release 256:79-91. https ://doi.org/10.1016/j.jconrel.2017.04.012

Allahyari M, Mohabati R, Amiri S, Rastaghi ARE, Babaie J, Mahdavi M, Vatanara A, Golkar M (2016) Synergistic effect of rSAG1 and rGRA2 antigens formulated in PLGA microspheres in eliciting immune protection against Toxoplasama gondii. Exp Parasitol 170:236-246. https://doi.org/10.1016/j.exppara.2016.09.008

Anderson JM, Shive MS (2012) Biodegradation and biocompatibility of PLA and PLGA microspheres. Adv Drug Deliv Rev 64:72-82. https://doi.org/10.1016/S0169-409X(97)00048-3

Basu A, Kunduru KR, Doppalapudi S, Domb AJ, Khan W (2016) Poly(lactic acid) based hydrogels. Adv Drug Deliv Rev 107:192205. https://doi.org/10.1016/j.addr.2016.07.004

Brady JM, Cutright DE, Miller RA, Battistone GC, Hunsuck EE (1973) Resorption rate, route of elimination, and ultrastructure of the implant site of polylactic acid in the abdominal wall of the rat. $\mathrm{J}$ Biomed Mater Res Part A 7(2):155-166. https://doi.org/10.1002/ jbm.820070204

Bruno C, Agnolon V, Berti F, Bufali S, O’Hagan DT, Baudner BC (2016) The preparation and characterization of PLG nanoparticles with an entrapped synthetic TLR7 agonist and their preclinical evaluation as adjuvant for an adsorbed DTaP vaccine. Eur J Pharm Biopharm 105:1-8. https://doi.org/10.1016/j.ejpb.2016.05.013

Cai H, Liang Z, Huang W, Wen L, Chen G (2017) Engineering PLGA nano-based systems through understanding the influence of nanoparticle properties and cell-penetrating peptides for cochlear drug delivery. Int J Pharm 532(1):55-65. https://doi.org/10.1016/j.ijpha rm.2017.08.084 
Carreño JM, Perez-Shibayama C, Gil-Cruz C, Printz A, Pastelin R, Isibasi A, Chariatte D, Tanoue Y, Lopez-Macias C, Gander B (2016) PLGA-microencapsulation protects Salmonella typhi outer membrane proteins from acidic degradation and increases their mucosal immunogenicity. Vaccine 34(35):4263-4269. https://doi. org/10.1016/j.vaccine.2016.05.036

Chong CS, Cao M, Wong WW, Fischer KP, Addison WR, Kwon GS, Tyrrell DL, Samuel J (2005) Enhancement of T helper type 1 immune responses against hepatitis B virus core antigen by PLGA nanoparticle vaccine delivery. J Controll Release 102(1):85-99. https://doi.org/10.1016/j.jconrel.2004.09.014

Coelho JF, Ferreira PC, Alves P, Cordeiro R, Fonseca AC, Góis JR, Gil MH (2010) Drug delivery systems: advanced technologies potentially applicable in personalized treatments. EPMA J 1(1):164209. https://doi.org/10.1007/s13167-010-0001-x

Cruz J, Flórez J, Torres R, Urquiza M, Gutiérrez J, Guzmán F, Ortiz C (2017) Antimicrobial activity of a new synthetic peptide loaded in polylactic acid or poly (lactic-co-glycolic) acid nanoparticles against Pseudomonas aeruginosa, Escherichia coli $\mathrm{O} 157$ : $\mathrm{H} 7$ and methicillin resistant Staphylococcus aureus (MRSA). Nanotechnology 28(13):135102. https://doi.org/10.1088/1361-6528/aa5f63

Dalzon B, Lebas C, Jimenez G, Gutjahr A, Terrat C, Exposito J-Y, Verrier B, Lethias C (2016) Poly (Lactic Acid) nanoparticles targeting $\alpha 5 \beta 1$ integrin as vaccine delivery vehicle, a prospective study. PLoS ONE 11(12):e0167663. https://doi.org/10.1371/journ al.pone. 0167663

Danhier F, Ansorena E, Silva JM, Coco R, Le Breton A, Préat V (2012) PLGA-based nanoparticles: an overview of biomedical applications. J Controll Release 161(2):505-522. https://doi. org/10.1016/j.jconrel.2012.01.043

Dhakal S, Hiremath J, Bondra K, Lakshmanappa YS, Shyu D-L, Ouyang K, K-i Kang, Binjawadagi B, Goodman J, Tabynov K (2017) Biodegradable nanoparticle delivery of inactivated swine influenza virus vaccine provides heterologous cell-mediated immune response in pigs. J Controll Release 247:194-205. https://doi. org/10.1016/j.jconrel.2016.12.039

Du X, Wang J-L, Iqbal S, Li H, Cao Z, Wang Y-C, Du J, Wang J (2018) The effect of surface charge on oral absorption of polymeric nanoparticles. Biomater Sci 6(3):642-650. https://doi.org/10.1039/ c7bm01096f

Ekanem EE, Zhang Z, Vladisavljevic GT (2017a) Facile production of biodegradable bipolymer patchy and patchy Janus particles with controlled morphology by microfluidic routes. Langmuir 33(34):8476-8482. https://doi.org/10.1021/acs.langmuir.7b02506

Ekanem EE, Zhang Z, Vladisavljević GT (2017b) Facile microfluidic production of composite polymer core-shell microcapsules and crescent-shaped microparticles. J Colloid Interface Sci 498:387394. https://doi.org/10.1016/j.jcis.2017.03.067

El-Hammadi MM, Delgado ÁV, Melguizo C, Prados JC, Arias JL (2017) Folic acid-decorated and PEGylated PLGA nanoparticles for improving the antitumour activity of 5-fluorouracil. Int J Pharm 516(1):61-70. https://doi.org/10.1016/j.ijpha rm.2016.11.012

Fröhlich E (2012) The role of surface charge in cellular uptake and cytotoxicity of medical nanoparticles. Int J Nanomed 7:5577. https ://doi.org/10.2147/IJN.S36111

Gagliardi M, Bertero A, Bifone A (2017) Molecularly imprinted biodegradable nanoparticles. Sci Rep 7:40046. https://doi.org/10.1038/ srep40046

Genta I, Colonna C, Conti B, Caliceti P, Salmaso S, Speziale P, Pietrocola G, Chiesa E, Modena T, Dorati R (2016) CNA-loaded PLGA nanoparticles improve humoral response against $\mathrm{S}$. aureus-mediated infections in a mouse model: subcutaneous vs. nasal administration strategy. J Microencapsul 33(8):750-762

Gourdon B, Chemin C, Moreau A, Arnauld T, Baumy P, Cisternino S, Péan J-M, Declèves X (2017) Functionalized PLA-PEG nanoparticles targeting intestinal transporter PepT1 for oral delivery of acyclovir. Int J Pharm 529(1-2):357-370. https://doi. org/10.1016/j.ijpharm.2017.07.024

Gregory AE, Titball R, Williamson D (2013) Vaccine delivery using nanoparticles. Front Cell Infect Microbiol 3:13. https://doi. org/10.3389/fcimb.2013.00013

Gutierro I, Hernandez R, Igartua M, Gascon A, Pedraz J (2002) Size dependent immune response after subcutaneous, oral and intranasal administration of BSA loaded nanospheres. Vaccine 21(1):67-77. https://doi.org/10.1016/S0264-410X(02)00435-8

Gutjahr A, Phelip C, Coolen A-L, Monge C, Boisgard A-S, Paul S, Verrier B (2016) Biodegradable polymeric nanoparticles-based vaccine adjuvants for lymph nodes targeting. Vaccines 4(4):34. https://doi.org/10.3390/vaccines4040034

Hillaireau H, Couvreur P (2009) Nanocarriers' entry into the cell: relevance to drug delivery. Cell Mol Life Sci 66(17):2873-2896. https://doi.org/10.1007/s00018-009-0053-z

Hiremath J, K-i Kang, Xia M, Elaish M, Binjawadagi B, Ouyang K, Dhakal S, Arcos J, Torrelles JB, Jiang X (2016) Entrapment of H1N1 influenza virus derived conserved peptides in PLGA nanoparticles enhances $\mathrm{T}$ cell response and vaccine efficacy in pigs. PLoS ONE 11(4):e0151922. https://doi.org/10.1371/journ al.pone. 0151922

James R, Manoukian OS, Kumbar SG (2016) Poly (lactic acid) for delivery of bioactive macromolecules. Adv Drug Deliv Rev 107:277-288. https://doi.org/10.1016/j.addr.2016.06.009

Johansen P, Men Y, Merkle HP, Gander B (2000) Revisiting PLA/ PLGA microspheres: an analysis of their potential in parenteral vaccination. Eur J Pharm Biopharm 50(1):129-146. https://doi. org/10.1016/S0939-6411(00)00079-5

Kasturi SP, Kozlowski PA, Nakaya HI, Burger MC, Russo P, Pham M, Kovalenkov Y, Silveira EL, Havenar-Daughton C, Burton SL (2017) Adjuvanting a simian immunodeficiency virus vaccine with toll-like receptor ligands encapsulated in nanoparticles induces persistent antibody responses and enhanced protection in TRIM5 $\alpha$ restrictive macaques. J Virol 91(4):e01844. https://doi. org/10.1128/JVI.01844-16

Kumar R, Jha D, Panda AK (2019) Polylactide/polylactide-co-glycolide-based delivery system for bioactive compounds against microbes. Pharm Microbes. https://doi.org/10.1007/978-3-03001881-8_3

Li P, Asokanathan C, Liu F, Khaing KK, Kmiec D, Wei X, Song B, Xing D, Kong D (2016) PLGA nano/micro particles encapsulated with pertussis toxoid (PTd) enhances Th1/Th17 immune response in a murine model. Int J Pharm 513(1):183-190. https ://doi.org/10.1016/j.ijpharm.2016.08.059

Liu L, Ma P, Wang H, Zhang C, Sun H, Wang C, Song C, Leng X, Kong D, Ma G (2016) Immune responses to vaccines delivered by encapsulation into and/or adsorption onto cationic lipid-PLGA hybrid nanoparticles. J Controll Release 225:230-239. https://doi. org/10.1016/j.jconrel.2016.01.050

Marasini N, Khalil ZG, Giddam AK, Ghaffar KA, Hussein WM, Capon RJ, Batzloff MR, Good MF, Skwarczynski M, Toth I (2016) Lipid core peptide/poly (lactic-co-glycolic acid) as a highly potent intranasal vaccine delivery system against Group A streptococcus. Int J Pharm 513(1):410-420. https://doi.org/10.1016/j.ijpha rm.2016.09.057

Margaroni M, Agallou M, Kontonikola K, Karidi K, Kammona O, Kiparissides C, Gaitanaki C, Karagouni E (2016) PLGA nanoparticles modified with a TNF $\alpha$ mimicking peptide, soluble Leishmania antigens and MPLA induce $\mathrm{T}$ cell priming in vitro via dendritic cell functional differentiation. Eur J Pharm Biopharm 105:18-31. https://doi.org/10.1016/j.ejpb.2016.05.018

Metz SW, Tian S, Hoekstra G, Yi X, Stone M, Horvath K, Miley MJ, DeSimone J, Luft CJ, de Silva AM (2016) Precisely molded nanoparticle displaying DENV-E proteins induces 
robust serotype-specific neutralizing antibody responses. PLoS Neg1 Trop Dis 10(10):e0005071. https://doi.org/10.1371/journ al.pntd.0005071

Mitragotri S, Burke PA, Langer R (2014) Overcoming the challenges in administering biopharmaceuticals: formulation and delivery strategies. Nat Rev Drug Discov 13(9):655. https://doi.org/10.1038/ $\operatorname{nrd} 4363$

Murariu M, Dubois P (2016) PLA composites: from production to properties. Adv Drug Deliv Rev 107:17-46. https://doi. org/10.1016/j.addr.2016.04.003

Nabi H, Rashid I, Ahmad N, Durrani A, Akbar H, Islam S, Bajwa AA, Shehzad W, Ashraf K, Imran N (2017) Induction of specific humoral immune response in mice immunized with ROP18 nanospheres from Toxoplasma gondii. Parasitol Res 116(1):359-370. https://doi.org/10.1007/s0043

Oyewumi MO, Kumar A, Cui Z (2010) Nano-microparticles as immune adjuvants: correlating particle sizes and the resultant immune responses. Expert Rev Vaccines 9(9):1095-1107. https ://doi.org/10.1586/erv.10.89

Pagels RF, Prud'homme RK (2015) Polymeric nanoparticles and microparticles for the delivery of peptides, biologics, and soluble therapeutics. J Controll Release 219:519-535. https://doi. org/10.1016/j.jconrel.2015.09.001

Pandey SK, Patel DK, Maurya AK, Thakur R, Mishra DP, Vinayak M, Haldar C, Maiti P (2016) Controlled release of drug and better bioavailability using poly (lactic acid-co-glycolic acid) nanoparticles. Int J Biol Macromol 89:99-110. https://doi.org/10.1016/j. ijbiomac.2016.04.065

Panyam J, Labhasetwar V (2003) Dynamics of endocytosis and exocytosis of poly (D, L-lactide-co-glycolide) nanoparticles in vascular smooth muscle cells. Pharm Res 20(2):212-220. https://doi. org/10.1023/A:1022219003551

Panyam J, Zhou W-Z, Prabha S, Sahoo SK, Labhasetwar V (2002) Rapid endo-lysosomal escape of poly (D, L-lactide-co-glycolide) nanoparticles: implications for drug and gene delivery. FASEB J 16(10):1217-1226. https://doi.org/10.1096/fj.02-0088com

Pei Y, Mohamed MF, Seleem MN, Yeo Y (2017) Particle engineering for intracellular delivery of vancomycin to methicillin-resistant Staphylococcus aureus (MRSA)-infected macrophages. J Controll Release 267:133. https://doi.org/10.1016/j.jconrel.2017.08.007

Peres C, Matos AI, Conniot J, Sainz V, Zupančič E, Silva JM, Graça L, Gaspar RS, Préat V, Florindo HF (2017) Poly (lactic acid)based particulate systems are promising tools for immune modulation. Acta Biomater 48:41-57. https://doi.org/10.1016/j.actbi o.2016.11.012

Peyre M, Audran R, Estevez F, Corradin G, Gander B, Sesardic D, Johansen P (2004) Childhood and malaria vaccines combined in biodegradable microspheres produce immunity with synergistic interactions. J Controll Release 99(3):345-355. https://doi. org/10.1016/j.jconrel.2004.07.014

Qu S, Zhao L, Zhu J, Wang C, Dai C, Guo H, Hao Z (2017) Preparation and testing of cefquinome-loaded poly lactic-co-glycolic acid microspheres for lung targeting. Drug Deliv 24(1):745-751. https ://doi.org/10.1080/10717544.2017.1321058

Raudszus B, Mulac D, Langer K (2018) A new preparation strategy for surface modified PLA nanoparticles to enhance uptake by endothelial cells. Int J Pharm 536(1):211-221. https://doi. org/10.1016/j.ijpharm.2017.11.047

Roointan A, Kianpour S, Memari F, Gandomani M, Gheibi Hayat SM, Mohammadi-Samani S (2018) Poly (lactic-co-glycolic acid): the most ardent and flexible candidate in biomedicine! Int J Polym Mater Polym Biomater 67:1028. https://doi.org/10.1080/00914 037.2017.1405350

Roopngam P, Liu K, Mei L, Zheng Y, Zhu X, Tsai H-I, Huang L (2016) Hepatitis C virus E2 protein encapsulation into poly d, 1-lactic-co-glycolide microspheres could induce mice cytotoxic
T-cell response. Int J Nanomed 11:5361. https://doi.org/10.2147/ IJN.S109081

Saffer EM, Tew GN, Bhatia SR (2011) Poly (lactic acid)-poly (ethylene oxide) block copolymers: new directions in self-assembly and biomedical applications. Curr Med Chem 18(36):5676-5686. https:// doi.org/10.2174/092986711798347324

Sahin A, Esendagli G, Yerlikaya F, Caban-Toktas S, Yoyen-Ermis D, Horzum U, Aktas Y, Khan M, Couvreur P, Capan Y (2017) A small variation in average particle size of PLGA nanoparticles prepared by nanoprecipitation leads to considerable change in nanoparticles' characteristics and efficacy of intracellular delivery. Artif Cells Nanomed Biotechnol 45:1657. https://doi. org/10.1080/21691401.2016.1276924

Saraiva C, Praça C, Ferreira R, Santos T, Ferreira L, Bernardino L (2016) Nanoparticle-mediated brain drug delivery: overcoming blood-brain barrier to treat neurodegenerative diseases. J Controll Release 235:34-47. https://doi.org/10.1016/j.jconrel.2016.05.044

Seok H, Noh JY, Lee DY, Kim SJ, Song CS, Kim YC (2017) Effective humoral immune response from a H1N1 DNA vaccine delivered to the skin by microneedles coated with PLGA-based cationic nanoparticles. J Controll Release 265:66-74. https://doi. org/10.1016/j.jconrel.2017.04.027

Shah RR, O'Hagan DT, Amiji MM, Brito LA (2014) The impact of size on particulate vaccine adjuvants. Nanomedicine 9(17):26712681. https://doi.org/10.2217/nnm.14.193

Silva A, Soema P, Slütter B, Ossendorp F, Jiskoot W (2016) PLGA particulate delivery systems for subunit vaccines: linking particle properties to immunogenicity. Hum Vaccines Immunother 12(4):1056-1069. https://doi.org/10.1080/21645515.2015.1117714

Steinbach JM, Seo Y-E, Saltzman WM (2016) Cell penetrating peptide-modified poly (lactic-co-glycolic acid) nanoparticles with enhanced cell internalization. Acta Biomater 30:49-61. https:// doi.org/10.1016/j.actbio.2015.11.029

Suk JS, Xu Q, Kim N, Hanes J, Ensign LM (2016) PEGylation as a strategy for improving nanoparticle-based drug and gene delivery. Adv Drug Deliv Rev 99:28-51. https://doi.org/10.1016/j. addr.2015.09.012

Sun Z, Yan X, Liu Y, Huang L, Kong C, Qu X, Wang M, Gao R, Qin H (2017) Application of dual targeting drug delivery system for the improvement of anti-glioma efficacy of doxorubicin. Oncotarget 8(35):58823. https://doi.org/10.18632/oncotarget.19221

Tan Z, Liu W, Liu H, Li C, Zhang Y, Meng X, Tang T, Xi T, Xing Y (2017) Oral Helicobacter pylori vaccine-encapsulated acidresistant HP55/PLGA nanoparticles promote immune protection. Eur J Pharm Biopharm 111:33-43. https://doi.org/10.1016/j. ejpb.2016.11.007

Thomas N, Thorn C, Richter K, Thierry B, Prestidge C (2016) Efficacy of poly-lactic-co-glycolic acid micro-and nanoparticles of ciprofloxacin against bacterial biofilms. J Pharm Sci 105(10):31153122. https://doi.org/10.1016/j.xphs.2016.06.022

Tran TH, Tran TTP, Nguyen HT, Dai Phung C, Jeong J-H, Stenzel MH, Jin SG, Yong CS, Truong DH, Kim JO (2018) Nanoparticles for dendritic cell-based immunotherapy. Int J Pharm. https://doi. org/10.1016/j.ijpharm.2018.03.029

Türeli NG, Torge A, Juntke J, Schwarz BC, Schneider-Daum N, Türeli AE, Lehr C-M, Schneider M (2017) Ciprofloxacin-loaded PLGA nanoparticles against cystic fibrosis $P$. aeruginosa lung infections. Eur J Pharm Biopharm 117:363-371. https://doi.org/10.1016/j. ejpb.2017.04.032

Tzeng SY, Guarecuco R, McHugh KJ, Rose S, Rosenberg EM, Zeng Y, Langer R, Jaklenec A (2016) Thermostabilization of inactivated polio vaccine in PLGA-based microspheres for pulsatile release. J Controll Release 233:101-113. https://doi.org/10.1016/j.jconr el.2016.05.012 
Vasir JK, Labhasetwar V (2007) Biodegradable nanoparticles for cytosolic delivery of therapeutics. Adv Drug Deliv Rev 59(8):718728. https://doi.org/10.1016/j.addr.2007.06.003

Wan F, Yang M (2016) Design of PLGA-based depot delivery systems for biopharmaceuticals prepared by spray drying. Int J Pharm 498(1):82-95. https://doi.org/10.1016/j.ijpharm.2015.12.025

Wang Q, Barry MA, Seid CA, Hudspeth EM, McAtee CP, Heffernan MJ (2017) 3M-052 as an adjuvant for a PLGA microparticle-based Leishmania donovani recombinant protein vaccine. J Biomed Mater Res B Appl Biomater 106:1587. https://doi. org/10.1002/jbm.b.33965

Watkins HC, Pagan CL, Childs HR, Posada S, Chau A, Rios J, Guarino C, DeLisa MP, Whittaker GR, Putnam D (2017) A single dose and long lasting vaccine against pandemic influenza through the controlled release of a heterospecies tandem M2 sequence embedded within detoxified bacterial outer membrane vesicles. Vaccine 35:5373. https://doi.org/10.1016/j.vaccine.2017.08.013

Yang HW, Ye L, Guo XD, Yang C, Compans RW, Prausnitz MR (2017) Ebola vaccination using a DNA vaccine coated on PLGA-PLL/ $\gamma$ PGA Nanoparticles administered using a microneedle patch. Adv Healthc Mater 6(1):1600750. https://doi.org/10.1002/adhm.20160 0750

Zhang N-Z, Xu Y, Wang M, Chen J, Huang S-Y, Gao Q, Zhu X-Q (2016) Vaccination with Toxoplasma gondii calcium-dependent protein kinase 6 and rhoptry protein 18 encapsulated in poly (lactide-co-glycolide) microspheres induces long-term protective immunity in mice. BMC Infect Dis 16(1):168. https://doi. org/10.1186/s12879-016-1496-0

Zhao F, Zhao Y, Liu Y, Chang X, Chen C, Zhao Y (2011) Cellular uptake, intracellular trafficking, and cytotoxicity of nanomaterials. Small 7(10):1322-1337. https://doi.org/10.1002/smll.201100001

Publisher's Note Springer Nature remains neutral with regard to jurisdictional claims in published maps and institutional affiliations. 